\title{
EL TRATAMIENTO DE LOS PHRASAL VERBSEN LOS MANUALES DE INGLÉS LENGUA EXTRANJERA
}

Andrea Artusi ${ }^{1}$

Universitat de València

Recibido 24/02/2020 Aceptado 12/05/2020

En este artículo nos proponemos investigar el tratamiento de los phrasal verbs ingleses en una muestra representativa de manuales de inglés de Educación Secundaria y Bachillerato, con el objeto de analizar, desde el punto de vista tanto cuantitativo como cualitativo: (i) si se nombran explícitamente en el índice de los manuales; (ii) si existen apartados teóricos específicos donde se expliquen sus características sintácticas y semánticas (y de ser así, en qué consisten); (iii) si se ofrecen actividades y ejercicios concebidos para practicar e interiorizar dichas estructuras y a qué enfoque metodológico atienden; si se observan diferencias en el tratamiento de los PV en general (iv) y dependiendo del nivel lingüístico de referencia de cada manual (v).

In this article we will study how phrasal verbs (PhVs) are dealt with in a representative sample of EFL student's books of Secondary Education and Baccalaureate. From both a quantitative and qualitative point of view, the following aspects will be analysed: (i) whether PhVs are explicitly included into the index of each manual; (ii) whether there are any specific theoretical sections explaining their syntactic and semantic characteristics (and if so, what they consist of); (iii) whether there are any activities and/or exercises designed to practice and internalize these structures and, if any, to which methodological approach they attend; if any differences in the treatment of the PhVs may be observed both in general (iv), and/or depending on the corresponding linguistic level of each manual (v).

\section{DOI}

https://doi.org/10.15366/didacticas2020.22.002

\section{PALABRAs Clave}

Phrasal verbs; glotodidáctica; inglés lengua extranjera; manuales de inglés LE. 


\section{INTRODUCCIÓN: LA IMPORTANCIA DE LOS PHRASAL VERBS INGLESES Y LOS ESCASOS RESULTADOS COSECHADOS HASTA HOY}

Cualquier persona que haya estudiado inglés como lengua extranjera (LE) es consciente de que, al escuchar un programa radiofónico en inglés, así como al ver una película o una serie de televisión anglosajona en versión original, o bien al leer un libro o una página web en esta lengua, los hablantes no nativos nos enfrentamos a menudo con estructuras verbales del tipo give up, make out, get over with, cuya comprensión, en ocasiones, nos puede resultar muy complicada. Ello se debe a que, estas construcciones verbales, comúnmente denominadas phrasal verbs (de ahora en adelante, PV), a pesar de estar constituidas por más de una palabra, -en particular, un verbo $(\mathrm{V})$ y una partícula adverbial $(\mathrm{P})$ - tienden a presentar un significado unitario (Carter y McCarthy, 2006, pp. 429-431), que, además, a veces es no composicional y semánticamente opaco, esto es, no deducible de la suma de los significados (literales) de sus elementos constitutivos (por ejemplo, give up, literalmente «dar arriba», significa 'rendirse').

Por otra parte, para el discente de inglés las dificultades vinculadas al empleo de estas construcciones trascienden el ámbito semántico, pues también pueden darse problemas a la hora de aprender su correcta configuración sintáctica, debido a las numerosas restricciones distribucionales a las que están supeditadas a nivel oracional (Claridge 2000, p. 5154; Quirk et al. 1985, p. 1152-1160).

En el caso de los hispanohablantes, además, familiarizarse con estas construcciones constituye todo un desafío lingüístico, dada la escasez de estructuras parecidas en el español actual (Calvo Rigual, 2008) ${ }^{1}$, por lo que la posibilidad de que se den transferencias positivas (transfers) entre la L1 y la LE resulta prácticamente nula. De hecho, en la actualidad, en las producciones tanto orales como escritas de los hispanohablantes se observa un empleo muy reducido de PV: incluso los alumnos que cuentan con un nivel avanzado de inglés utilizan estas estructuras con una frecuencia notablemente inferior con respecto a los hablantes nativos, en concreto, un 44,5\% menos (Weibel, 2007, p. 77).

El interés de los PV estriba en su frecuencia de uso y en su productividad, sin lugar a dudas muy elevadas en los hablantes nativos de inglés. Debido a su uso extendido, de hecho, los PV han sido estudiados en el ámbito de la Lingüística atendiendo a enfoques metodológicos diversos, entre otros, la Gramática Generativa, la Gramática Cognitiva y la Tipología Lingüística (Cappelle, 2015), también de carácter aplicado, en cuanto a la didáctica de las lenguas extranjeras (Condon, 2008; Garnier y Schmitt, 2016; Rudzka-Ostyn, 2003). Además, la productividad de estas estructuras en la lengua inglesa ha sido corroborada en los estudios de la Lingüística de Corpus, en los cuales se ha señalado que los PV

\footnotetext{
${ }^{1}$ En Calvo Rigual (2008) se proporciona un listado de 49 PV españoles extraídos de un corpus lingüístico lexicográfico, un número de construcciones muy exiguo si lo comparamos con los miles de PV existentes en inglés. Además, las estructuras halladas en español en pocas ocasiones presentan características particularmente destacables y un comportamiento homogéneo (Calvo Rigual, 2008, p. 63), por lo que no sería posible afirmar que en castellano estas estructuras pertenecen a una nueva categoría verbal.
} 
constituyen un tercio del inventario verbal del inglés (Li et al., 2003, p. 513) ${ }^{2}$, y que, si se consideran las estructuras y el léxico de lengua inglesa en su conjunto, de cada 9,6 palabras, una de ellas constituye un PV, lo que significa, dicho de otra manera, que normalmente se hallan un promedio de dos PV en cada página redactada en inglés (Gardner y Davies, 2007, p. 347) ${ }^{3}$.

\section{OBJETIVOS Y METODOLOGÍA}

Precisamente debido a la envergadura del fenómeno lingüístico, así como a las dificultades que acarrean su comprensión y su empleo activo por parte de los discentes, en este artículo pretendemos analizar de qué manera se aborda la enseñanza de los PV ingleses en las clases de Educación Secundaria y de Bachillerato en España. Para hacerlo, nos ocuparemos de analizar un corpus constituido por 24 libros de texto de inglés LE, que en la actualidad se comercializan en todo el territorio nacional español y que en muchas ocasiones han sido concebidos especificadamente para un público hispanohablante. Consideramos que el corpus seleccionado es una muestra representativa de los manuales con los que se suele trabajar en Educación Secundaria y Bachillerato en España en la actualidad, puesto que, al seleccionar el material hemos procurado asegurar la máxima representatividad en función, por una parte, del número de editoriales (cinco) y de autores (veinticinco), y por otra, de los niveles lingüísticos (da A2 a B2) (ocho manuales por cada nivel).

En nuestra investigación, pretendemos llevar a cabo un estudio tanto cuantitativo como cualitativo de dichos manuales, con el propósito de determinar:

i. si en el índice de los manuales se nombra el fenómeno lingüístico que nos ocupa, o bien si se suele obviar;

ii. si existen apartados teóricos específicos donde se expliquen sus características sintácticas y semánticas (y de ser así, en qué consisten);

iii. si se ofrecen actividades y ejercicios concebidos para practicar e interiorizar dichas estructuras, a qué enfoque metodológico atienden - de entre las numerosas metodologías aplicadas a la enseñanza de los $\mathrm{PhVs}$ que han ido surgiendo a lo largo de las últimas décadas (Aldahesh, 2013, pp. 90-107)- y en qué consisten;

iv. si se observan diferencias en el tratamiento de los PV dependiendo del nivel lingüístico de referencia de cada manual;

\footnotetext{
2 En el estudio citado se ha contrastado material procedente de dos corpus de tipo lexicográfico, en particular, dos diccionarios de PV y otros "machine-readable dictionaries" (Li et al., 2003, p. 513).

${ }^{3}$ Datos recabados del vaciado del British National Corpus (100 millones de palabras).
} 
v. qué similitudes y qué diferencias principales se hacen patentes en los manuales analizados en lo que respecta al tratamiento de estas construcciones.

En lo que atañe a la metodología, hemos adoptado una perspectiva afín a la de estudios anteriores sobre las expresiones multipalabra (Artusi, 2017; López-Jiménez, 2013; Stanič, 2014). Así, además de observar si los PV se mencionan explícitamente en el índice de cada manual y si se explican sus características sintácticas, semánticas y fonológicas en su interior (objetivos i y ii), para el objetivo (iii) de nuestro trabajo, hemos atendido a un enfoque adoptado en un estudio anterior acerca del tratamiento de las multi-word lexical units en 24 libros de texto de inglés y español LE (López-Jiménez, 2013, p. 340). En particular, con el fin de analizar los ejercicios y actividades presentes en los manuales para enseñar los PV, nos hemos servido de la siguiente clasificación, en la que se diferencia entre:

a) "ejercicio mecánico", que puede llevarse a cabo sin que sea necesaria la comprensión explícita de la estructura, y en el que solo hay una respuesta correcta. Por ejemplo, pertenecen a esta categoría los ejercicios en los que hay que completar una unidad léxica añadiendo una vocal o una consonante.

b) “ejercicio cerrado". En este caso se precisa un mayor grado de comprensión de la construcción, y una vez más, solo puede haber una respuesta correcta, como en los cloze tests por excelencia: los denominados fill-in-the-gaps exercises.

c) “actividades abiertas", en las que los alumnos deben entender el valor semántico de la construcción y elegir entre diferentes opciones, teniendo en cuenta que más de una puede ser válida. En dichas actividades, además, podría haber un "vacío informativo" (explícito o implícito) entre los interlocutores (es decir, no todos los participantes en la actividad disponen de toda la información), para evitar que el intercambio resulte artificial.

d) "actividad comunicativa": requiere una respuesta abierta y/o la elección de una unidad léxica (en nuestro caso, un PV) necesaria para poder llevar a cabo la actividad. Aquí también se observa disparidad informativa entre los interlocutores. Los discentes han de interactuar entre ellos para conseguir un resultado final, esto es, alcanzar un objetivo que es imposible de lograr de manera individual (como, por ejemplo, la redacción de una campaña publicitaria por parejas utilizando los PV proporcionados).

e) “ejercicio y actividad ambiguos”, que contiene, a la vez, más de una característica perteneciente a las categorías anteriores.

Por otro lado, para poder conseguir los objetivos (iv) y (v) de nuestra investigación hemos cruzado los datos obtenidos de la clasificación recién mencionada con los niveles 
de pertenencia de los manuales (iv) y hemos comparado los datos cualitativos y cuantitativos de nuestro estudio entre los diferentes manuales del corpus (v).

\section{RESULTADOS}

\section{Los PV en el índice de los manuales}

Nos parece particularmente interesante observar la presencia o la ausencia de los PV en los índices de los manuales, no solo porque nos permite averiguar en qué niveles se empiezan a enseñar de manera explícita a los discentes, sino también para determinar qué etiqueta metalingüística se suele emplear para referirse a las construcciones que nos ocupan y en qué apartados de los manuales suelen figurar.

En el $75 \%$ de los casos en el índice se habla de forma explícita de phrasal verbs, la denominación comúnmente reconocida para referirse a estas construcciones. Por el contrario, en el restante $25 \%$ de los manuales no se hace mención alguna de los PV. El hecho de que los PV sean destacados en gran parte de los índices del corpus nos confirma, una vez más, la importancia de dichas estructuras en la lengua inglesa.

$\mathrm{Al}$ observar los niveles lingüísticos, es fácil percatarse de que los PV son ignorados en el índice de los libros correspondientes a los niveles más bajos de entre los considerados. En particular, los PV no se mencionan en cinco manuales de A2/+ y en un manual del nivel B1. ${ }^{4}$ En otras palabras, en cinco de los seis manuales de nivel A2 se ha decidido no hacer referencia a los PV. A primera vista, todo ello parece responder a una postura tradicional (aparentemente generalizada), según la cual no se deberían enseñar estas construcciones (al igual que las colocaciones, las expresiones idiomáticas y las palabras compuestas) en los niveles iniciales de una lengua extranjera, sin que haya pruebas empíricas fehacientes que justifiquen dicha postura metodológica (López-Jiménez, 2013, p. 343).

En cambio, al examinar más de cerca el contenido de los manuales que no mencionan en su índice los PV, nos damos cuenta de que en realidad es erróneo pensar que en el nivel A2 se ignoran por completo, pues, en su interior, sí que se enseñan algunos (como get up, wake up, grow up). En ocasiones, los Student's Books lo hacen sin ofrecer ninguna explicación sobre las propiedades de las construcciones ni pedir que se realice ningún ejercicio o actividad con ellos, mientras que, en otras, los PV constituyen la construcción lingüística que se pretende practicar a través de alguna actividad, como ocurre en Mosaic 2 (p. 50, ejercicio 1), en el que se ofrece un listado de PV con sentido composicional (en los que la $\mathrm{P}$ expresa la dirección del movimiento codificado por $\mathrm{V}$ ) para que los discentes los asocien a las figuras correspondientes que se hallan al final de la página.

Por otra parte, si consideramos los índices que contemplan los PV, cabe decir que en todos y cada uno de ellos las construcciones que nos atañen se introducen en la sección

\footnotetext{
${ }^{4}$ En Succeed in English 2, curiosamente, a pesar de no hacerse referencia explícita a los PV como categoría verbal, se incluye en la sección vocabulary el PV growing up.
} 
vocabulary, excepto en Out and About 1 y 2 , donde, en cambio, se dedica una columna del índice aparte a los PV que se abordan en las distintas unidades, y en la que se señalan, además, las diferentes posibilidades distribucionales del objeto directo (OD) en las combinaciones transitivas (check out sth / sb o check sth / sb out) (Out and About 2, p. 3). Además, otros dos manuales - Golden Experience B2 y Mosaic 4- ofrecen información acerca de las características de los PV tratados, al hablar de PV inseparables y separables y de PV transitivos e intransitivos, respectivamente.

Para añadir, en el índice de tan solo cuatro manuales, se suelen asociar los PV a un campo semántico determinado (por ejemplo, en Mosaic 3, se señala "phrasal verbs: relationships" o "phrasal verbs and nouns: free-time activities", p. 2).

En Prepare 3 (p. 3), se observa una falta de homogeneidad en el tratamiento de estas construcciones, ya que, dentro del mismo índice, en primer lugar, se menciona un PV en la sección vocabulary sin denominarlo con el término metalingüístico correspondiente (phrasal verbs), y poco más tarde, por el contrario, se hace referencia explícita a los PV.

En resumidas cuentas, en cuanto a los índices del corpus analizado, si bien en su mayoría se hace referencia explícita a los PV, se observa una falta de homogeneidad a la hora de señalarlos, puesto que:

- se han hallado diferencias entre los diferentes manuales en lo que atañe al tratamiento de los PV mencionados, ya que, en ocasiones, se subrayan las características sintácticas, mientras que, en otras, no;

- $\quad$ solo en un número exiguo de ocasiones los PV se relacionan con un campo semántico determinado;

- dentro de un mismo nivel lingüístico, si bien en la mayor parte de los manuales se mencionan en el apartado vocabulary, en otros, se crea una columna específica para destacarlos;

- incluso dentro de un mismo manual, se han encontrado diferencias en el tratamiento de algunos PV, a pesar de que pertenecen a la misma categoría verbal.

\section{Apartados teóricos acerca de los PV}

A continuación, analizamos los apartados de los manuales que aportan algún tipo de información, sugerencia, definición, o explicación gramatical sobre los PV, esto es, examinamos en qué medida se procura llevar a cabo aportaciones teóricas que detallen las características del fenómeno lingüístico con el objeto de facilitar su aprendizaje.

En 19 manuales de 24 no se da ninguna explicación teórica. En los otros casos, además, la reflexión teórica alrededor de los PV es mínima. En particular, se observa que:

- $\quad$ en Pulse 3(B1, p.13), se ofrece una definición de phrasal verb (“a phrasal verb consists of a root verb and a particle (adverb or preposition). The meaning is different 
from the verb on its own"), sin aportar ningún otro tipo de información (como, por ejemplo, la diferencia entre PV transitivos e intransitivos);

- en Key to Bachillerato 2 (B2, p. 109), se hace hincapié, con un simple comentario, en la importancia de la $\mathrm{P}$ en el seno de la construcción desde el punto de vista semántico ("When learning phrasal verbs, try to understand the meaning of the particle, e. g. up can mean begin (take up, set up)"). Sin embargo, tal vez por falta de espacio, no se explican los diferentes significados que suelen asociarse a dicha $\mathrm{P}$ (up), sino que los autores se limitan a ofrecer un ejemplo con valor aspectual incoativo.

- En Gold Experience B1 (p. 56) se dan sugerencias al discente sobre cómo memorizar los PV. A nuestra manera de ver, la explicación parece poco clara, debido a la falta de contextualización, por lo que nos parece lógico cuestionar su utilidad ("Try learning them in pairs! By preposition: sort out $\rightarrow$ find out, By verb: take place $\rightarrow$ take part in; by opposites: take up $\rightarrow$ give up").

- en Key to Bachillerato 1 (B1+, p. 136) (Figura 16) y Gold Experience (B2, p. 68), en cambio, se explica brevemente la diferencia entre PV separables e inseparables, y se señala la distribución de los elementos oracionales en presencia de un pronombre.

En definitiva, parece cuanto menos sorprendente que en tan solo dos manuales se expliquen las características sintácticas principales de los PV, sobre todo si pensamos en las restricciones distribucionales a las que están supeditadas estas construcciones a nivel oracional, sin lugar a dudas de difícil adquisición por parte de los hablantes de inglés LE. Además, el hecho de no ofrecer ninguna explicación de tipo teórico no parece estar relacionado al nivel lingüístico, ya que en los niveles más altos entre los considerados (B1 y B2), por lo general, tampoco se contempla la presencia de aclaraciones teóricas.

\section{Ejercicios y actividades sobre los PV}

Al llevar a cabo la clasificación de las actividades y los ejercicios concebidos para practicar e interiorizar los PV (véase § 2), hemos obtenido los siguientes resultados (Figura 1).

Tal y como se puede observar en la Figura (1), en nuestro corpus, el $81 \%$ de los ejercicios concebidos para aprender los PV constituyen lo que hemos denominado "ejercicio cerrado", es decir, un tipo de ejercicio que precisa un cierto grado de comprensión de la construcción para que pueda ser realizado por el discente, y en el cual solo puede haber una respuesta correcta. Esta clase de ejercicios se manifiesta en los manuales de diferentes maneras. Entre las principales, destacamos los ejercicios en los que el discente debe: 
- ante un listado de PV y una serie de imágenes, asociar cada PV con su imagen correspondiente;

- ante un listado de PV y un listado de significados (explicación del valor semántico de los PV, a modo de glosa explicativa), combinar cada PV con su significado correspondiente;

- $\quad$ rellenar los espacios vacíos que se hallan en una frase (el tradicional fill-in-the-gaps exercise), bien con un $\mathrm{PV}$, bien con la $\mathrm{P}$ adecuada para que la construcción tenga sentido en la oración. En todo caso se proporciona al discente un listado de opciones entre las que ha de elegir.

- ante un listado de PV y un listado de oraciones, reescribir cada oración mediante la sustitución del verbo principal por el PV adecuado al contexto oracional.

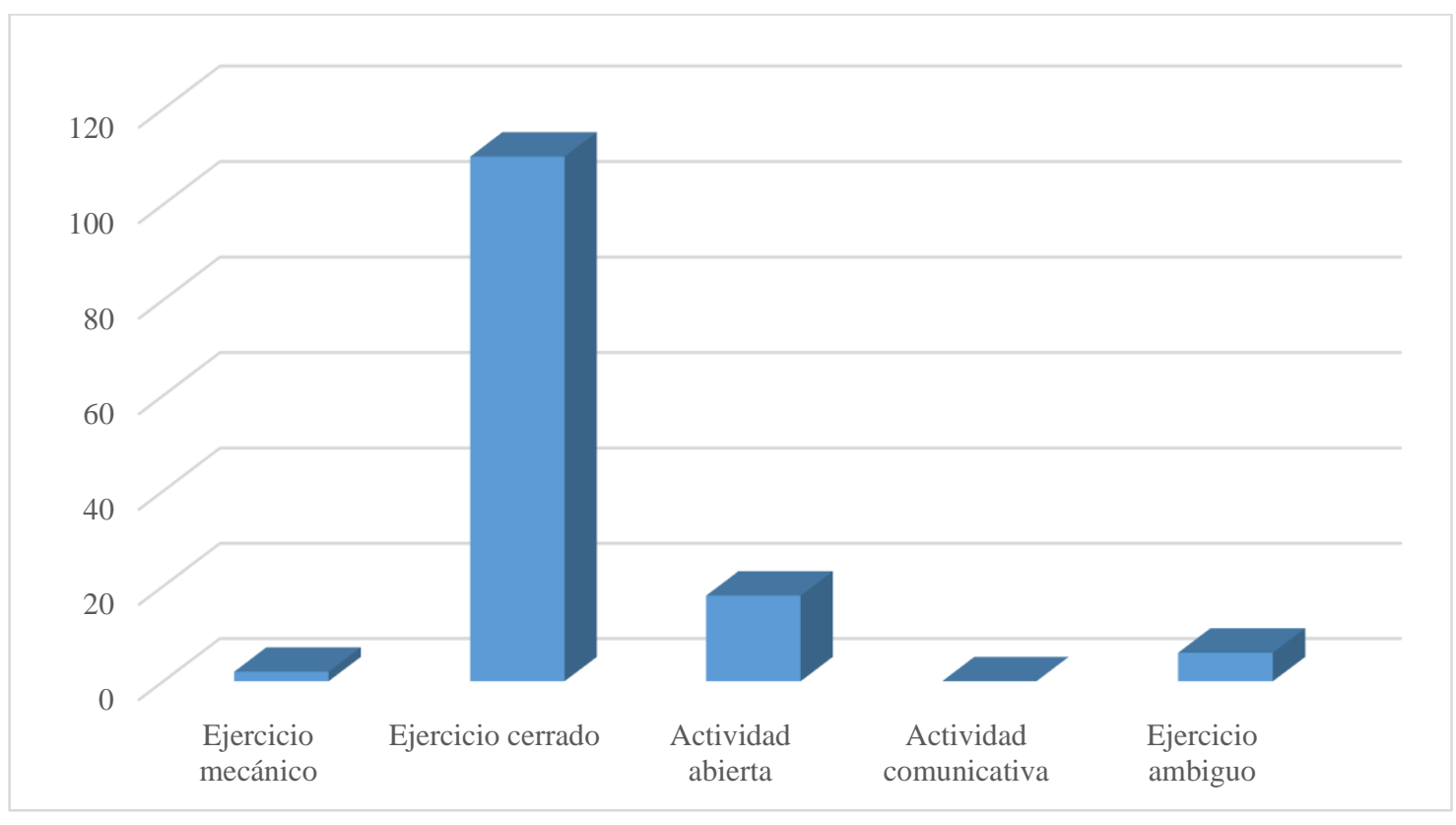

Figura 1: Ejercicios y actividades sobre los PV en el corpus

Además, con un número exiguo de casos, en esta categoría aparecen también otros tipos de ejercicios, en los que se le pide al discente que:

- $\quad$ ante una lista de PV y un listado de significados antónimos, asocie cada PV con el antónimo correspondiente;

- cambie la P perteneciente a un PV de la oración, por la P adecuada.

Por otro lado, en el 13\% de los casos los manuales proponen "actividades abiertas", esto es, actividades en las que los alumnos han de entender el significado de la construcción y 
elegir entre diferentes opciones, teniendo en cuenta que más de una puede ser válida. Así pues, se pide a los discentes que trabajen por parejas y que den lugar a un diálogo haciendo uso de los PV previamente aprendidos en la unidad. A modo de ejemplo, en Key to Bachillerato $1(\mathrm{~B} 1+$, p. 53) se ofrece a los alumnos un texto en el que han de rellenar los espacios en blanco con un PV o una colocación de los proporcionados en un recuadro. Hasta este momento, se recurre a lo que hemos llamado "ejercicio cerrado". Más adelante en la misma página, en cambio, los alumnos deben crear un diálogo (a modo de entrevista, del tipo pregunta/respuesta, para que expresen su opinión sobre cuestiones controvertidas) y así poner en práctica las estructuras expuestas en el texto anterior (PV y colocaciones). En estos casos, dependiendo de la contestación que decidan dar al compañero, podrán seleccionar y utilizar un PV u otro de los presentes en el texto, sin seguir necesariamente el mismo orden en el que figuran originariamente.

En lo que atañe a los "ejercicios ambiguos", en el corpus analizado son poco frecuentes (4\%). Es posible incluir en esta categoría los ejercicios que piden al alumno que se invente unas oraciones (característica que comparte con las actividades abiertas) utilizando uno de los PV de la unidad. Dicha libertad de elección de las estructuras, sin embargo, no es una condición suficiente para que se pueda considerar como un ejercicio perteneciente al enfoque comunicativo. De hecho, entre otros factores, la interacción entre el alumnado en estos casos resulta nula.

En cuanto a los "ejercicios mecánicos", en nuestro corpus solo hemos encontrado dos ejemplos, por ende, no muy representativos desde el punto de vista estadístico (2\%) y tipológico, en los que el discente debe, a partir del infinitivo del PV get up escribir la forma del gerundio y del pasado de la construcción. En ambos casos, para poder llevar a cabo el ejercicio el alumno no tiene que entender, necesariamente, el significado de la construcción y tampoco la ha de utilizar activamente en un contexto de uso determinado.

Las actividades de tipo comunicativo, por su parte, brillan por su ausencia, pues en ningún manual del corpus analizado hemos hallado ejemplos de actividades comunicativas que puedan fomentar el dominio de las construcciones que nos ocupan.

En definitiva, en lo que concierne a los ejercicios y actividades, el enfoque metodológico que predomina en los manuales de Educación Secundaria y Bachillerato es de carácter tradicional. Como hemos observado, en efecto, a los discentes simplemente se les pide que asocien un PV determinado con su significado, o bien que rellene unos espacios en blanco, al seleccionar un PV de una lista de posibles opciones. El empleo de estas construcciones por parte del alumnado resulta artificial, a menudo descontextualizado, ajeno a la vida cotidiana de quien estudia inglés como LE y, por consiguiente, poco interesante y aburrido.

\section{Los PV según la competencia lingüística}

Si consideramos el tratamiento de los PV en nuestro corpus en función de los tres niveles de competencia lingüística, obtenemos los datos a continuación (Tabla 1)5. Por lo general, 
es evidente que los niveles con mayor número de ejercicios y actividades sobre los PV son el B1 y el B2, mientras que el A2, se ocupa de estas construcciones analíticas con una frecuencia equivalente a la mitad de la que presenta en los otros niveles.

\begin{tabular}{|c|c|c|c|c|c|}
\hline NIVEL & TIPO 1 & TIPO 2 & TIPO 3 & TIPO 4 & TIPO 5 \\
\hline A2 & 2 & 18 & 5 & 0 & 1 \\
\hline B1 & 0 & 42 & 8 & 0 & 5 \\
\hline B2 & 0 & 50 & 5 & 0 & 0 \\
\hline TOTAL & 2 & 110 & 18 & 0 & 6 \\
\hline
\end{tabular}

Tabla 1: Los PV según el nivel de competencia

Todo ello parece responder a la voluntad de los autores de los manuales de limitar el tratamiento de las expresiones multipalabra (colocaciones, expresiones idiomáticas, palabras compuestas y PV) lo más posible en los niveles de competencia más bajos, para abordar estas construcciones de manera progresiva a medida que el dominio lingüístico del discente vaya aumentando. Sin embargo, tal y como hemos afirmado anteriormente, a día de hoy no existen pruebas empíricas que sustenten dicha postura metodológica, pues aún no ha sido demostrado que reservar su aprendizaje para los niveles avanzados puede conllevar mejores resultados.

En cuanto a las diferencias detectables entre los tres niveles analizados, los PV que se pretenden enseñar en la competencia lingüística del A2 constituyen, en muchas ocasiones, construcciones básicas para describir actividades cotidianas (wake up, get up), mientras que en los niveles B1 y B2 el abanico de estructuras y de campos semánticos tratados es más amplio. Sin embargo, también encontramos ejemplos de otros tipos de PV en el nivel A2, como en Gold Experience A2 (p. 108), incluso con valor semántico más opaco, como look out ('tener cuidado').

Por otra parte, en el análisis cualitativo de los niveles B1 y B2 no se han hallado diferencias relevantes que puedan estar relacionadas con la competencia lingüística, ya que la manera de tratar los PV y los ejercicios y actividades propuestos son del todo parecidos.

\section{Análisis de los manuales del corpus}

Desde el punto de vista cuantitativo, los datos (Tabla 2) muestran que hay una gran disparidad en el tratamiento de los PV. En términos absolutos, esto es, si consideramos el conjunto del corpus, hay manuales que cuentan con numerosos ejercicios y actividades sobre

\footnotetext{
${ }^{5}$ Las cinco categorías de la tabla (tipo 1-5) se corresponden con la clasificación metodológica resumida en $\S 2$.
} 
los PV (hasta un máximo de 17), y otros que, en cambio, presentan de 0 a 2 ejercicios en total. Además, esta discrepancia se da también dentro de un mismo nivel de competencia lingüística: en el caso del nivel A2, por ejemplo, se va de manuales con 8 ejercicios sobre los PV, hasta otros que cuentan con tan solo dos, o incluso ninguno.

\begin{tabular}{|c|c|c|c|c|c|c|c|}
\hline MANUAL & NIVEL & TIPO 1 & TIPO 2 & TIPO 3 & TIPO 4 & TIPO 5 & TOT. \\
\hline Gold Experience A2 & A2 & 0 & 4 & 4 & 0 & 0 & 8 \\
\hline Gold Experience B1 & B1 & 0 & 6 & 2 & 0 & 2 & 10 \\
\hline Gold Experience B2 & B2 & 0 & 1 & 0 & 0 & 0 & 1 \\
\hline Key to Bachillerato 1 & $\mathrm{~B} 1+$ & 0 & 13 & 3 & 0 & 1 & 17 \\
\hline Key to Bachillerato 2 & B2 & 0 & 8 & 1 & 0 & 0 & 9 \\
\hline Mosaic 2 & A2 & 0 & 4 & 0 & 0 & 0 & 4 \\
\hline Mosaic 3 & $\mathrm{~A} 2+$ & 0 & 3 & 0 & 0 & 0 & 3 \\
\hline Mosaic 4 & B1 & 0 & 5 & 1 & 0 & 1 & 7 \\
\hline Out and About 1 & $\mathrm{~B} 1+$ & 0 & 2 & 0 & 0 & 0 & 2 \\
\hline Out and About 2 & B2 & 0 & 2 & 0 & 0 & 0 & 2 \\
\hline Prepare Student's book level 2 (A2) & $\mathrm{A} 2$ & 0 & 2 & 0 & 0 & 0 & 2 \\
\hline Prepare Student's book level 3 (A2) & $A 2$ & 0 & 3 & 0 & 0 & 0 & 3 \\
\hline Prepare Student's book level 4 (BT) & B1 & 0 & 8 & 0 & 0 & 0 & 8 \\
\hline Prepare Student's book level 5 (BT) & B1 & 0 & 3 & 2 & 0 & 0 & 5 \\
\hline Pulse 1 & A2 & 0 & 0 & 0 & 0 & 0 & 0 \\
\hline Pulse 2 & $A 2+$ & 0 & 0 & 0 & 0 & 0 & 0 \\
\hline Pulse 3 & B1 & 0 & 2 & 0 & 0 & 0 & 2 \\
\hline Succeed in English 1 & $A 2$ & 2 & 2 & 1 & 0 & 1 & 6 \\
\hline Succeed in English 2 & B1 & 0 & 3 & 0 & 0 & 1 & 4 \\
\hline Succeed in English 3 & B2 & 0 & 7 & 1 & 0 & 0 & 8 \\
\hline Succeed in English 4 & $\mathrm{~B} 2+$ & 0 & 8 & 2 & 0 & 0 & 10 \\
\hline Upgrade 1 & B2 & 0 & 7 & 0 & 0 & 0 & 7 \\
\hline Upgrade 2 & $\mathrm{~B} 2+$ & 0 & 7 & 1 & 0 & 0 & 8 \\
\hline Viewpoints 2 & $\mathrm{~B} 2+$ & 0 & 10 & 0 & 0 & 0 & 15 \\
\hline
\end{tabular}

Tabla 2: Análisis cuantitativo de los manuales 
Por otro lado, al analizar cualitativamente el corpus, se percibe cierta falta de homogeneidad en el tratamiento de los PV en lo que respecta a qué características de estas construcciones deben aprenderse. Para ejemplificar, solo dos manuales - Pulse 3 (p. 13, ejercicio 2) y Mosaic 4 (p. 65, ejercicio 6)- hacen hincapié en cómo han de pronunciarse y proponen ejercicios en los que los discentes puedan colocar el acento primario y secundario correctamente (cf. Pérez Alonso, 2001, pp. 517-518). Todo ello se añade, además, a las consideraciones que ya hemos llevado a cabo acerca de la inclusión de apartados teóricos sobre los PV, por lo general inexistentes, aunque con algunas excepciones, que no hacen más que confirmar la disparidad del tratamiento reservado a estas estructuras.

\section{CONCLUSIONES}

Tras analizar el tratamiento de los PV en un corpus de 24 manuales de inglés LE que se utilizan en la actualidad en las clases de Educación Secundaria y Bachillerato en España, nuestra investigación ha destacado los resultados siguientes:

- los PV se mencionan de forma explícita en el índice del 75\% de los manuales - casi siempre en el apartado vocabulary-, lo que nos confirma que las construcciones analíticas verbales que nos ocupan juegan un papel primordial en la didáctica del inglés;

- la denominación que se utiliza para referirse a este fenómeno lingüístico es phrasal verb, frente a otras propuestas en la bibliografía, como, por ejemplo, verb-particle constructions, en los estudios de Tipología Lingüística (Talmy, 1985, 2000);

- si bien gran parte de los manuales incluyen los PV en su índice, en estas primeras páginas se observa cierta falta de homogeneidad en su tratamiento, no solo entre los manuales pertenecientes a un mismo nivel de competencia, sino también, en ocasiones, en el seno de un mismo manual;

- en 19 manuales de 24 no se da ninguna explicación teórica acerca de las características sintácticas o semánticas de los PV. Es más, en tan solo dos de ellos se explica la diferencia entre PV separables e inseparables, mientras que, en los restantes, los autores se limitan a proporcionar alguna sugerencia para su memorización. Además, el nivel de competencia lingüística de cada manual parece no haber influido en la inclusión de posibles explicaciones teóricas;

- el $81 \%$ de los ejercicios que se ofrecen para practicar e interiorizar los PV atienden a un enfoque metodológico tradicional, según el cual los discentes han de asociar PV con imágenes o con glosas explicativas de su significado, o bien realizar ejercicios del tipo fill-in-the-gaps, donde la participación activa en la manipulación y el procesamiento del material lingüístico por parte del alumnado es nula; 
- aunque el 13\% de las actividades propuestas pueden considerarse más abiertas, en el sentido de que los alumnos pueden seleccionar qué PV utilizar para llevarlas a cabo (conscientes de que más de una opción de las que disponen puede ser válida), no se ha hallado ninguna actividad de tipo comunicativo. Por otra parte, los ejemplos de "ejercicios mecánicos” y "ambiguos” parecen ser poco relevantes desde el punto de vista estadístico y tipológico;

- al contrastar los datos recabados sobre el análisis de cada manual, se aprecia una disparidad cuantitativa y cualitativa en el tratamiento de los PV, tanto si se considera el corpus en su conjunto, como dentro de cada nivel de competencia lingüística.

Los resultados que acabamos de resumir ponen de manifiesta la falta de homogeneidad en la didáctica de los PV en los manuales del corpus, así como la adopción de un enfoque metodológico tradicional, según el cual el aprendizaje del léxico se fomenta a través de actividades cerradas, con escasa implicación y bajo nivel de procesamiento por parte de los discentes.

En conclusión, como es sabido, los enfoques metodológicos tradicionales en la didáctica de la lengua, que según nuestro estudio siguen empleándose de forma significativa, no han conllevado buenos resultados en cuanto al dominio de los PV por parte de los discentes. Por tanto, creemos en la necesidad de superar las metodologías más tradicionales a favor de un cambio de paradigma de carácter comunicativo, que pueda reflejarse en el diseño de los ejercicios y actividades propuestos en los manuales. Si bien no tenemos la certeza de que la adopción de metodologías más innovadoras comporte mejoras en este sentido, opinamos que, sin lugar a dudas, vale la pena asumir este reto.

\section{REFERENCIAS}

ALDAHESH, A. Y. (2013). Teaching English Phrasal Verbs to Non-native speakers of English, Arab World English Journal, 4(1), 90-107.

ARTUSI, A. (2017). Il trattamento dei verbi sintagmatici nelle classi di italiano L2. Una proposta metodologica. En: G. Caprara y G. Marangon (Eds.), Italiano e Dintorni. La realtà linguistica italiana: approfondimenti di didattica, variazione e traduzione (pp. 393-413). Fráncfort del Meno: Peter Lang.

CALVO RIGUAL, C. (2008). I verbi sintagmatici italiani con appunti contrastivi con lo spagnolo e il catalano. En C. González y P. Mogorrón. (Eds.), Estudios y análisis de fraseología contrastiva: Lexicografía y Traducción (pp. 47-66). Alicante, España: Universidad de Alicante. 
CAPPELLE, B. (2005). Particle Patterns in English. Lovaina, Bélgica: Katholieke Universiteit Leuven.

CARTER, R. Y MCCARTHY M. (2006). Cambdridge Grammar of English. Cambridge, Reino Unido: Cambridge University Press.

CLARIDGE, C. (2000). Multi-word Verbs in Early Modern English. A Corpus-based Study. Amsterdam-Atlanta, Holanda-Estados Unidos: Rodopi.

CONDON, N. (2008). How cognitive linguistic motivations influence the learning of phrasal verbs. En F. BOERS Y S. LINDSTROMBERG (Eds.), Applications of Cognitive Linguistics: Cognitive Linguistics approaches to Teaching Vocabulary and Phraseology (pp. 133-158). Berlín, Alemania: Mouton de Gruyter.

GARDNER, D. Y DAVIES, M. (2007). Pointing Out Frequent Phrasal Verbs: A CorpusBased Analysis, TESOL QUARTERLY, 41(2), 339-359.

GARNIER, M. Y SCHMITT, N. (2016). 'Picking up' polysemous phrasal verbs: How many do learners know and what facilitates this knowledge? System, 59, 29-44.

LI, W., ZHANG X., NIU C., JIANG Y. Y SRIHARI R. (2003). An Expert Lexicon Approach to Identifying English Phrasal Verbs. En E. W. HINRICHS Y D. ROTH. (Eds.), Proceedings of the 41st Annual Meeting of the Association for Computational Linguistics (pp. 513- 520). Stroudsburg (PA), Estados Unidos: Association for Computational Linguistics.

LÓPEZ-JIMÉNEZ, M. D. (2013). Multi-word lexical units in L2 textbooks. Resla, 26, 333348.

QUIRK, R., GREENBAUM S., LEECH G. Y SVARTVIK J. (1985). A Comprehensive Grammar of the English Language. Londres, Reino Unido: Longman.

PÉREZ ALONSO, Ma A. (Ed.) (2001). Diccionario Oxford de Phrasal Verbs inglés-español. Oxford/New York, Reino Unido/Estados Unidos: Oxford University Press.

RUDZKA-OSTYN, B. (2003). Word power: Phrasal verbs and compounds: A cognitive approach. Nueva York, Estados Unidos: Walter de Gruyter.

STANIČ, D. (2014). Le unità fraseologiche nei libri di testo d'italiano come lingua straniera; Analisi di libri di testo graduati secondo i livelli di competenza linguistica del Quadro comune europeo di riferimento per la conoscenza delle lingue (QCER). Linguistica, 54, 167-188.

TALMY, L. (1985). Lexicalization patterns semantic structure in lexical forms. En T. Shopen (Ed.), Language Typology and Syntactic Description, Vol. III. Grammatical Categories and the Lexicon (pp. 57-149). Cambridge, Reino Unido: Cambridge University Press.

TALMY, L. (2000). Toward a Cognitive Semantics: Typology and Process in Concept Structuring. Cambridge, Reino Unido: MIT Press, vol. 2. 
WAIBEL, B. (2007). Phrasal verbs in learner English: A corpus-based study of German and Italian students (Tesis doctoral). Albert-Ludwigs-Universität, Friburgo (Alemania).

\section{Manuales analizados}

ALEVIZOS, K. Y GAYNOR S. (2014). Gold Experience A2 Key for Schools Students' Book. Harlow, Reino Unido: Pearson Education Limited.

BARRACLOUGH, C. Y GAYNOR S. (2014). Gold Experience B1 Key for Schools Students' Book. Harlow, Reino Unido: Pearson Education Limited.

BRADFIELD, B. Y WETZ B. (2014). Succeed in English Student's Book 4, Vantage B2+. Oxford, Reino Unido: Oxford University Press.

CAPEL, A. Y JOSEPH N. (2015). Prepare! Student's Book Level 5. Cambridge, Reino Unido: Cambridge University Press.

CRAWFORD, M. (2014). Pulse 2 Student’s Book. Londres, Reino Unido: McMillan.

EDWARDS, L. Y STEPHENS M. (2014). Gold Experience B2 Key for Schools Students' Book. Harlow, Reino Unido: Pearson Education Limited.

GORMLEY, K. (2015). Mosaic Student's Book 4. Oxford, Reino Unido: Oxford University Press.

HANCOCK, M. Y MCDONALD A. (2015). Out \& About Student's Book 1 - Bachillerato, Batxillerat, Batxilergoa, Bachalerato. Cambridge, Reino Unido: Cambridge University Press.

HANCOCK, M. Y MCDONALD A. (2015). Out \& About Student's Book 2 - Bachillerato, Batxillerat, Batxilergoa, Bachalerato. Cambridge, Reino Unido: Cambridge University Press.

KELLY, P. (2015). Mosaic Student's Book 3. Oxford, Reino Unido: Oxford University Press.

KOSTA, J. Y WILLIAMS M. (2015). Prepare! Student's Book Level 2. Cambridge, Reino Unido: Cambridge University Press.

KOSTA, J. Y WILLIAMS M. (2015). Prepare! Student's Book Level 3. Cambridge, Reino Unido: Cambridge University Press.

MCBETH, C. (2013). Pulse 1 Student's Book. Londres, Reino Unido: McMillan.

MCBETH, C. (2014). Pulse 3 Student’s Book. Londres, Reino Unido: McMillan.

PELTERET, C. (2014). Mosaic Student's Book 2. Oxford, Reino Unido: Oxford University Press.

REILLY, P. Y NORRIS R. (2010). Upgrade Student's Book Bachillerato 1. Londres, Reino Unido: McMillan. 
REILLY, P. Y NORRIS R. (2010). Upgrade Student's Book Bachillerato 2. Londres, Reino Unido: McMillan.

RUBIO SANTANA, J. M. Y GRANT E. (2010). Viewpoints for Bachillerato 2. Nicosia, Chipre: Burlington Books.

STYRING, J. Y TIMS N. (2015). Prepare! Student's Book Level 4. Cambridge, Reino Unido: Cambridge University Press.

WETZ, B. Y GORMLEY K. (2014). Key to Bachillerato, Batxillerat, Batxilergoa, Bachalerato Student's Book 1. Oxford, Reino Unido: Oxford University Press.

WETZ, B. (2014). Key to Bachillerato, Batxillerat, Batxilergoa, Bachalerato Student's Book 2. Oxford, Reino Unido: Oxford University Press.

WETZ, B. (2013). Succeed in English Student's Book 1, Waystage A2. Oxford, Reino Unido: Oxford University Press.

WETZ, B. (2013). Succeed in English Student's Book 2, Threshold B1. Oxford, Reino Unido: Oxford University Press.

WETZ, B. (2013). Succeed in English Student's Book 3, Vantage B2. Oxford, Reino Unido: Oxford University Press. 